\title{
Modeling of Coil Pre-Stress Loss During Cool-Down in the Main Dipoles of the Large Hadron Collider
}

\author{
P. Ferracin, W. Scandale, E. Todesco, and D. Tommasini
}

\begin{abstract}
We describe a finite element mechanical model of the main LHC dipole, based on the geometry and on the properties of its components; coil characteristics are derived from measurements on stacks of conductors. We show how to define equivalent properties of cable blocks that take into account the collaring procedure when it is not explicitly modeled. Numerical results are then compared to experimental measurements of loads and deformations in dipole prototypes. At cryogenic temperature, equivalent properties are used to implement in the model a pressuredependent thermal contraction factor observed in stack measurements. This allows to forecast the large pre-stress loss during the cool-down observed in the LHC dipole prototypes.
\end{abstract}

Index Terms-Elastic modulus, mechanical properties, pre-stress losses, superconducting coil, thermal contraction.

\section{INTRODUCTION}

$\mathbf{T}$ HE MECHANICAL behavior of a superconducting magnet is an important feature to ensure good performance. Finite element models of the dipole cross-section are used both to optimize the mechanical structure, and to figure out the tolerances of the dipole components [1]-[3]. The modeling of these structures is not trivial [4]: this is mainly due to the complex behavior of the blocks of conductors, that feature a large mechanical hysteresis, a nonlinear stress-displacement relation, and difficulties in defining a thermal contraction factor [5]-[8].

The aim of this work is to predict coil deformations induced by loads during assembly and cool-down in the main dipoles of the Large Hadron Collider (LHC). In particular, we are interested in modeling stresses and displacements at ambient temperature and at cryogenic temperature, having as input the dimensions of the collared coil components. In [9] we evaluate the influence of the obtained deformations on the magnetic field-shape, showing that they are consistent with magnetic measurements and that they cannot be neglected for the LHC beam dynamics.

Here, we show how to build the mechanical model of the collared coil at $300 \mathrm{~K}$ and at $1.9 \mathrm{~K}$, based on the mechanical properties of stack of cables and on the collaring procedure. The model is validated by experimental measurements of the dependence of pre-stress and of collar deformations on the dimension of spacers between coil pole and collars (shims). The final cross-check is given by the comparison between simulations and experimental data of the azimuthal pre-stress loss in the coil from $300 \mathrm{~K}$ to $1.9 \mathrm{~K}$.

Manuscript received September 24, 2001

The authors are with the Main Magnets and Superconductors Group, LHC Division, CERN, 1211 Geneva 23, Switzerland (e-mail: \{paolo.ferracin: walter.scandale; ezio.todesco; davide.tommasini\}@cern.ch).

Publisher Item Identifier S 1051-8223(02)04270-7.

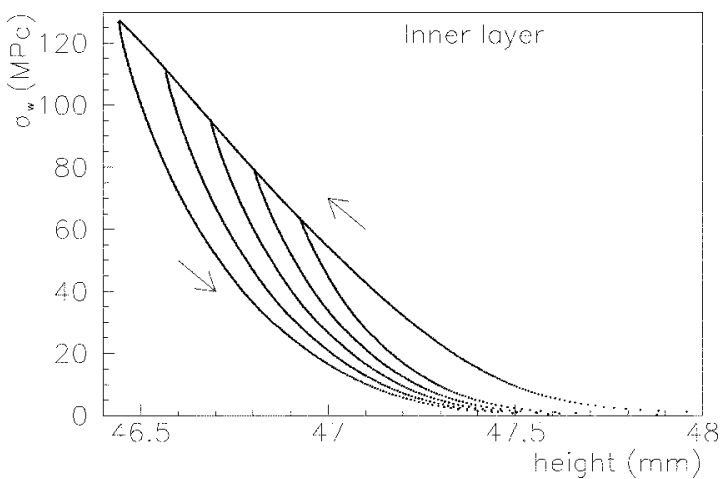

Fig. 1. Stress $\sigma_{w}$ (MPa) at ambient temperature versus total height of a stack of conductors for the inner layer, loading and unloading curves from different peak stresses (experimental data of [7]).

\section{Mechanical Behavior at $300 \mathrm{~K}$}

\section{A. Coil Mechanical Properties}

In [7] we presented measurements of the mechanical properties of the LHC main dipole coils. Stacks of conductors have been analyzed. As it has been observed in several types of insulated cables (see for instance [2]), the stack features a large elongation in a loading-unloading cycle. In Fig. 1 we plot the measured stress-displacement curves of a stack of the insulated cable of the inner layer. Five cycles of loading-unloading are shown, with peak stresses ranging from 70 to $120 \mathrm{MPa}$.

For a linear stress-strain relation, elastic modulus is the ratio between stress and strain. In the general case, it can be defined as the derivative of the stress with respect to the strain; therefore, it depends on the stress. Elastic modulus can be evaluated from the stress-displacement experimental curve shown in the above figure according to

$$
E(\sigma)=\frac{d \sigma}{d \epsilon}=\frac{d \sigma}{d l} l_{0}
$$

where $l_{0}$ is the stack height. One obtains values that considerably vary according to the pressure and to the cycle: between 40 and $100 \mathrm{MPa}$ the elastic modulus is around $5.5 \mathrm{GPa}$ in loading, and is between $6 \mathrm{GPa}$ and $18 \mathrm{GPa}$ in unloading (see Fig. 2).

\section{B. Collaring Procedure}

The large hysteresis shown in the stress-displacement plot is an important feature of our model: indeed, during the collaring, the coils are compressed up to a peak pre-stress and when the pressure is released only a fraction of it is left. This is due to the elasticity of the collars, that are pushed up and deformed by the pre-stress (the so-called spring-back). Moreover, to insert the collaring rods one also needs some clearance between the 


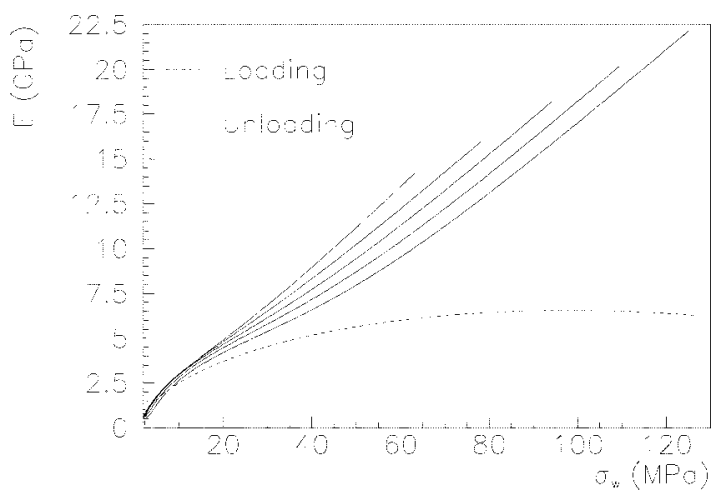

Fig. 2. Elastic modulus $E_{w},(\mathrm{GPa})$ versus stress $\sigma_{w}(\mathrm{MPa})$ at ambient temperature of a stack of conductors for the inner layer, loading and unloading curves from different peak stresses (experimental data).

holes and the rods that provokes an additional loss of pre-stress. Stress measurements made with capacitive gauges [10] placed on the coil poles have been taken for the LHC dipole prototypes; a ratio of 0.6 between the residual pre-stress after collaring and the peak pre-stress during collaring provides a good fit of experimental data.

\section{Modeling Coil Elasticity at $300 \mathrm{~K}$}

The aim of our finite element model is to compute loads and displacements, having in input the geometry of the components of the collared coil at $300 \mathrm{~K}$. Since the collaring procedure is not modeled explicitly and loads are applied through interferences of contact elements, the mechanical properties of the coil must include informations on the path followed in the stress-displacement graph (see Fig. 1). For instance, if the pole shims are larger than the nominal ones, during the collaring a higher peak pre-stress will be reached, and the unloading will take place along a different branch of the curve (see Fig. 3, solid lines). Therefore, the elastic modulus of the coil to be input in the model is neither the loading nor the unloading, but an "equivalent" modulus taking into account the peak pre-stress [11]. The equivalent stress-displacement curve is shown for the inner coil in Fig. 3 (dashed line). Its slope is smaller than both the unloading and the loading curve. Values for the loading, unloading and equivalent elastic moduli of the inner and outer coil are given in Table I.

\section{Results: Model versus Experiments}

The mechanical behavior foreseen by the model has been tested at $300 \mathrm{~K}$ with a dedicated experiment on a 1-m long prototype [11]. The magnet has been assembled five times with different pole spacer (shim) dimensions to vary the pre-stress and the azimuthal coil length in the collared coil. A "central" setting with nominal shims plus four other configurations with either inner or outer shims thicknesses different by $\pm 0.15 \mathrm{~mm}$ were tested. In each case, pre-stress at coil poles and vertical diameter of the collars were measured. Post-processing of experimental data show that a $0.1 \mathrm{~mm}$ thicker shim provokes a pre-stress increase of 12 to $13 \mathrm{MPa}$ on the corresponding layer. Results from the model are in agreement with experimental data (see Table II). Our model underestimates the outer layer pre-stress sensitivity of 2 to $4 \mathrm{MPa}$; this could be due to slight differences in the mechanical behavior between conductor stacks and

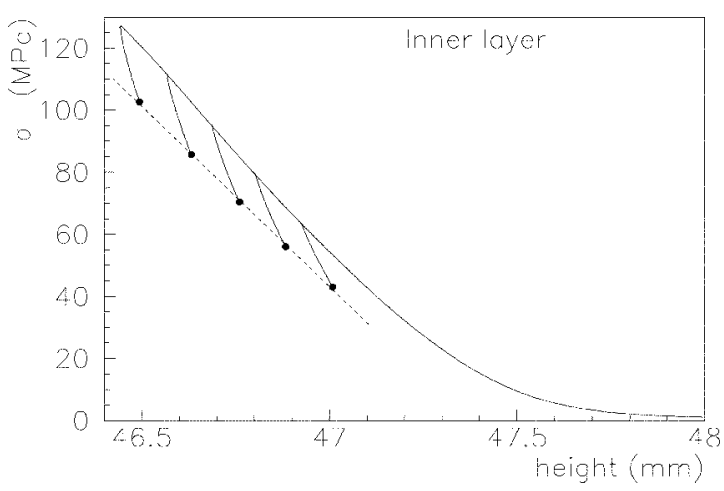

Fig. 3. Equivalent stress-displacement curve (dashed line) for the coil inner layer.

TABLE I

EQUIVALENT, LOADING AND UNLOADING ElASTIC MODULI (GPa) OF THE COIL AT $70 \mathrm{MPa}$

\begin{tabular}{lcc}
\hline \hline & Inner layer & Outer layer \\
\hline$E_{w}^{e}$ (equivalent) & 5.5 & 5.1 \\
$E_{w}^{u}$ (unloading) & 12.0 to 14.0 & 11.0 to 13.0 \\
$E_{w}^{l}$ (loading) & 6.6 & 6.4 \\
\hline \hline
\end{tabular}

TABLE II

Additional Pre-Stress (MPa) DUe to a 0.1 mm Thicker SHIM

\begin{tabular}{lcc}
\hline \hline & Inner layer & Outer layer \\
\hline Measurements & $12 \pm 1$ & $13 \pm 1$ \\
Finite element model & 12 & 10 \\
\hline \hline
\end{tabular}

conductor arcs. Indeed, one can point out that if the unloading elastic modulus at $70 \mathrm{MPa}$ (around $12 \mathrm{GPa}$ ) had been used, we would have obtained a sensitivity of $25 \mathrm{MPa}$.

Another ingredient necessary to obtain a correct mechanical model is the collar deformation due to the coil pre-stress. This is a combined effect of the elastic modulus of the collar material, i.e., austenitic steel $316 \mathrm{LN}$, and of the two-in-one collar geometry. The change in the collar vertical diameter due to a pre-stress variation of $10 \mathrm{MPa}$ on both layers was evaluated in the same experiment. The finite element model results give $0.036 \mathrm{~mm}$, in agreement with the experimental data of 0.037 $\pm 0.006 \mathrm{~mm}$.

\section{BehaVior AT 1.9 K AND PRe-Stress Loss}

\section{A. Coil Elasticity at $77 \mathrm{~K}$}

We measured the stress-displacement curves at $77 \mathrm{~K}$ for the inner and outer layer stacks; elastic moduli were worked out using the same scheme outlined for $300 \mathrm{~K}$ (see Section II-A). Results are shown in Fig. 4: the unloading modulus is similar to the ambient temperature case, whilst the loading modulus is increased by a factor 1.5 . For this reason, the difference between loading and unloading is less pronounced with respect to $300 \mathrm{~K}$, but the dependence of elastic modulus on pre-stress is still rather strong. These measurements have been used to model coil rigidity at $1.9 \mathrm{~K}$, since the variation of elasticity between $77 \mathrm{~K}$ and $1.9 \mathrm{~K}$ is negligible. 


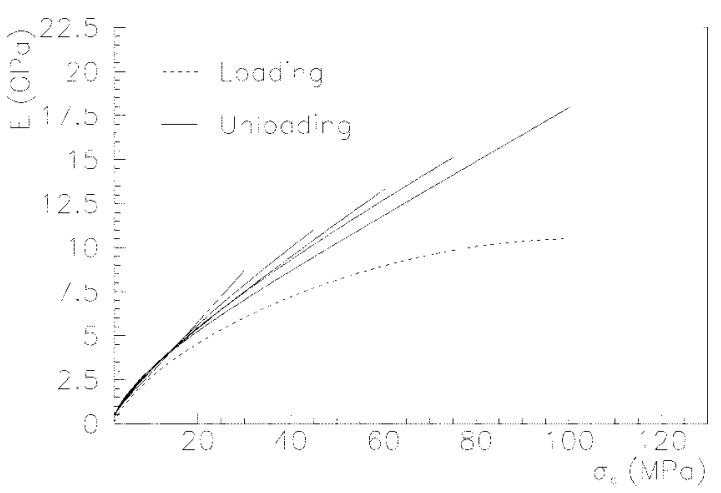

Fig. 4. Elastic modulus $E_{w}$ (GPa) versus stress $\sigma_{w}(\mathrm{MPa})$ at $77 \mathrm{~K}$ of a stack of conductors for the inner layer, loading and unloading curves from different peak stresses (experimental data).

\section{B. Pre-Stress Loss Equation}

Let us consider a conductor stack closed in a infinitely rigid mould, under a pre-stress $\sigma_{w}$ at $300 \mathrm{~K}$. If the whole system is cooled down at $1.9 \mathrm{~K}$, the difference in the coil deformation $\epsilon_{w}-\epsilon_{c}$ between $300 \mathrm{~K}$ and $1.9 \mathrm{~K}$ is equal to the difference in the integrated thermal contraction coefficients of the coil $\alpha_{b}$ and of the mould $\alpha_{c}$ :

$$
\epsilon_{w}-\epsilon_{c}=\alpha_{b}-\alpha_{c}
$$

Here, we neglect terms of the order of $\epsilon \alpha$ with respect to $\epsilon$ and $\alpha$. Using a linear relation between deformation and stresses $\sigma_{w}=E_{w} \epsilon_{w}$, and $\sigma_{c}=E_{c} \epsilon_{c}$, where $E_{w}$ and $E_{c}$ are the elastic moduli of the coil at $300 \mathrm{~K}$ and $1.9 \mathrm{~K}$ respectively, we obtain the equation for the pre-stress loss [4]

$$
\sigma_{c}=\frac{E_{c}}{E_{w}}\left(\sigma_{w}-E_{w}\left(\alpha_{b}-\alpha_{c}\right)\right)
$$

\section{Coil Integrated Thermal Contraction}

The pre-stress loss of a stack of cables in a closed mould is the usual technique to derive a measurement of the integrated thermal contraction of an insulated Rutherford cable [8]. A direct measurement of the thermal contraction is not possible, since the stack dimension when no load is applied shows a large indeterminacy. In [8], we measured five pre-stress losses of the inner and outer layer stacks, starting from different values of the pressure at room temperature $\sigma_{w}$. Results are shown in Table III.

Pre-stress losses $\left(\sigma_{w}, \sigma_{c}\right)$ provide through Eq. (3) the integrated thermal contraction $\alpha_{b}$ for a given choice of the elastic moduli $E_{w}$ and $E_{c}$. In our case at $300 \mathrm{~K}$ we use the equivalent elastic modulus $E_{w}^{e}$ defined in Section II (see Table I) to model the collaring. The elastic modulus at $77 \mathrm{~K}$ depends on the pressure (see Fig. 4). We use the elastic modulus at $\sigma_{c}=37$ $\mathrm{MPa}$, that is the pre-stress at $77 \mathrm{~K}$ reached by the stack from the nominal pre-stress at ambient temperature $\sigma_{w}=70 \mathrm{MPa}$ (see Table III). We consider the average between loading and unloading, choosing $\sigma_{c}=6.5 \mathrm{GPa}$ for both stacks. Using these elastic moduli, we can evaluate the integrated thermal contraction factor $\alpha_{b}$ through (3), with a small correction to take into account the mould cavity rigidity. We find a dependence of $\alpha_{b}$
TABLE III

Pre-STRESS LOSSES (MPa) From $300 \mathrm{~K}$ TO $77 \mathrm{~K}$ FOR INNER AND OUTER LAYER STACKS IN A CLOSED MOLD

\begin{tabular}{cccc}
\hline \hline$\sigma_{w}$ inner & $\sigma_{c}$ inner & $\sigma_{w}$ outer & $\sigma_{c}$ outer \\
\hline 80 & 45 & 80 & 43 \\
70 & 38 & 69 & 36 \\
60 & 34 & 61 & 29 \\
50 & 27 & 50 & 20 \\
40 & 18 & 40 & 14 \\
\hline \hline
\end{tabular}

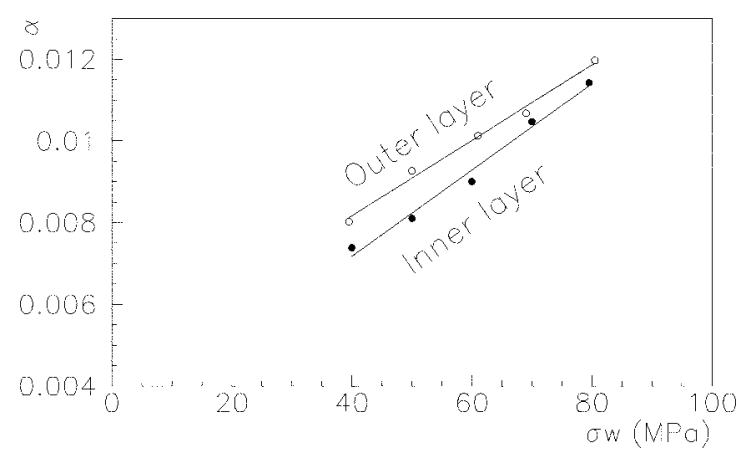

Fig. 5. Integrated thermal contraction coefficient from $300 \mathrm{~K}$ to $77 \mathrm{~K}$ versus pre-stress at $300 \mathrm{~K}$ and linear fit.

on the stress $\sigma_{w}$, obtaining results that range from 0.006 to 0.011 (see Fig. 5). A linear fit

$$
\alpha_{b}\left(\sigma_{w}\right)=\alpha_{b 0}+\alpha_{b 1} \sigma_{w}
$$

provides an analytic approximation of the experimental data. An increase of $10 \%$ in $\alpha_{b}$ has been applied to extrapolate these measurements from $77 \mathrm{~K}$ to $1.9 \mathrm{~K}$.

\section{Finite Element Modeling of Coil Elasticity at $1.9 \mathrm{~K}$ and Pre-Stress Loss}

The dependence of the thermal contraction factor on the prestress [see (4)] cannot be included in the finite element code ANSYS ${ }^{\mathrm{TM}}$ that has been used in simulations. Indeed, the prestress loss equation that includes a linear dependence of $\alpha$ on $\sigma_{w}$ for an infinitely rigid cavity is

$$
\sigma_{c}=\frac{E_{c}}{E_{w}^{e}}\left(\sigma_{w}-E_{w}^{e}\left(\alpha_{b 0}+\alpha_{b 1} \sigma_{w}-\alpha_{c}\right)\right) .
$$

One can group the dependence of $\alpha$ on $\sigma_{w}$ to the term that accounts for coil elasticity at cold

$$
\sigma_{c}=\frac{E_{c}}{E_{w}^{e}}\left(\sigma_{w}\left(1-\alpha_{b 1} E_{w}^{e}\right)-\left(\alpha_{b 0}-\alpha_{c}\right) E_{w}^{e}\right)
$$

The above equation can be cast in the form

$$
\sigma_{c}=\frac{E_{c}^{e}}{E_{w}^{e}}\left(\sigma_{w}-\left(\alpha_{b}^{e}-\alpha_{c}\right) E_{w}^{e}\right)
$$

where we defined a coil equivalent elastic modulus at $77 \mathrm{~K} E_{c}^{e}$ and an equivalent thermal contraction coefficient $\alpha_{b}^{e}$

$$
E_{c}^{e}=E_{c}\left(1-\alpha_{b 1} E_{w}^{e}\right) \quad \alpha_{b}^{e}=\alpha_{c}+\frac{\alpha_{b 0}-\alpha_{c}}{1-\alpha_{b 1} E_{w}^{e}}
$$


TABLE IV

Eouivalent THERmal CONTRACTION AND EQUiValent MOduli Defined IN (8) FOR THE INNER AND OUTER LAYER

\begin{tabular}{|c|c|c|}
\hline & Inner & Outer \\
\hline$E_{c}^{e}(\mathrm{GPa})$ & 2.7 & 3.0 \\
\hline$\alpha_{b}^{e}$ & 0.0040 & 0.0067 \\
\hline
\end{tabular}

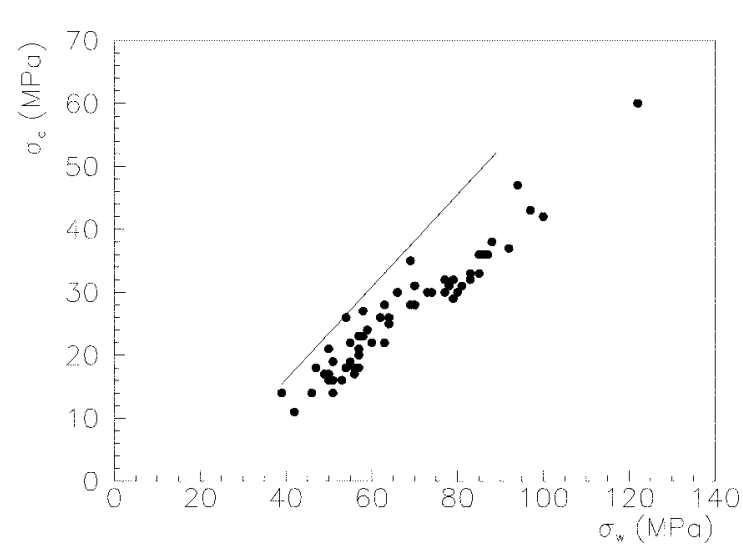

Fig. 6. Pre-stress at $1.9 \mathrm{~K}$ versus pre-stress after collaring at $300 \mathrm{~K}$ : experimental data (markers) versus analytical model (solid line).

Now the thermal contraction of the coil does not depend on the pressure and therefore can be used in the finite element code. The same formalism can be used without assuming an infinitely rigid coil cavity: numerical values derived for $\alpha_{b}^{e}$ and $E_{c}^{e}$ are given in Table IV. The analytical model with a stress-dependent $\alpha_{b}\left(\sigma_{w}\right)$ has been compared to the implementation in a finite element model using these equivalent properties, finding an excellent agreement. This model can be used to compute the initial state of the system when the magnet is powered at $1.9 \mathrm{~K}$. On the other hand, to compute deformations induced by electro-magnetic forces one has to use the measured stress-strain curves at $1.9 \mathrm{~K}$.

\section{E. Results: Model versus Experiments}

Several experimental results about pre-stress loss from collared coils at $300 \mathrm{~K}$ to cold mass at $1.9 \mathrm{~K}$ are available [4]. Data relative to both short and long prototypes are show in Fig. 6 (markers). Results from the analytical model based on Eq. (6) (plus the correction for the collar deformation) using the equivalent modulus at $300 \mathrm{~K}$ and the measured values of $E_{c}$ and $\alpha_{b}\left(\sigma_{w}\right)$ are shown in the same figure (solid line). The model overestimates the pre-stress at $1.9 \mathrm{~K}$ of 3 to $8 \mathrm{MPa}$ in the validity range of our linear approximation ( 40 to $80 \mathrm{MPa}$ at $300 \mathrm{~K}$ ). This can be compared to a dispersion of the data around the best fit of $5 \mathrm{MPa}$ (two sigma) in $\sigma_{c}$. This agreement can be considered satisfactory. We point out that a model that simply assumes a ratio $E_{c} / E_{w}$ equal to 1.5 (i.e., the usual hardening ratio) would predict a slope of the $\left(\sigma_{c}, \sigma_{w}\right)$ line of 1.5 , against a measured value of $0.53 \pm 0.10$, whilst our model gives a slope of 0.50 for the outer layer and of 0.60 for the inner layer.

\section{CONCLUSIONS}

We have presented a finite element model of the dipole cross-section the aim of which is to evaluate the dependence of coil loads and deformations on the dimensions of magnet components at $300 \mathrm{~K}$ and at $1.9 \mathrm{~K}$. The model is based on the measured mechanical and thermal properties of conductor stacks. Since the collaring procedure is not explicitly considered, we proposed to include it in the coil mechanical properties through the definition of equivalent elastic moduli. This also solves the ambiguity on the choice of the coil elastic modulus (loading or unloading) to be input in the model. We also propose coil equivalent properties at $1.9 \mathrm{~K}$ based on the measured stressdependence of the coil thermal contraction factor. Numerical results of the finite element model are compared to experimental data. Dependence of coil pre-stress and collar deformations on shim size show a good agreement with the model. We finally explain the large pre-stress loss from ambient to cryogenic temperature observed in experimental data.

\section{REFERENCES}

[1] M. S. Chapman, "A finite element analysis of an SSC dipole magnet (NC-9 cross-section)," in Supercollider 1. New York: Plenum Press, 1989, pp. 659-668.

[2] A. Devred et al., "About the mechanics of SSC dipole magnet prototypes," in AIP Conf. Proc., vol. 249, 1992, pp. 1309-1374.

[3] M. Bajko, P. Fessia, and D. Perini, "FEM computations concerning the effect of friction in two LHC main dipole structures," IEEE Trans. Appl. Superconduct., vol. 10, pp. 81-84, March 2000.

[4] P. Ferracin, W. Scandale, E. Todesco, P. Tropea, and I. Vanenkov, "Modeling thermo-mechanical structure of the LHC main dipole and influence on field quality," in Seventh European Particle Accelerator Conf., C. Petit-Jean Genaz, Ed.. New York, 2000, pp. 330-332.

[5] R. B. Meuser, S. Caspi, and W. S. Gilbert, "Measured mechanical properties of superconducting coil materials and their influence on coil prestress," IEEE Trans. Magn., vol. 17, pp. 2320-2323, Sept. 1981.

[6] D. R. Chichili, T. T. Arkan, J. P. Ozelis, and I. Terechkine, "Investigation of cable insulation and thermo-mechanical properties of epoxy impregnated $\mathrm{Nb}_{3} \mathrm{Sn}$ composites," IEEE Trans. Appl. Superconduct., vol. 10, pp. 1317-1320, Mar. 2000.

[7] P. Ferracin, E. Todesco, D. Tommasini, and W. Scandale, "Elastic modulus measurements of the LHC dipole superconducting coil at $300 \mathrm{~K}$ and at $77 \mathrm{~K}$," Adv. Cryog. Eng., vol. 47, to be published.

[8] - "Thermal contraction measurements of the superconducting coil of the main magnets for the large Hadron collider," Adv. Cryog. Eng., vol. 47 , to be published.

[9] _ _ "Impact of coil deformations on field quality in the large hadron collider main dipole," in MT-17 Conf..

[10] N. Siegel, D. Tommasini, and I. Vanenkov, "Design and use of capacitive force transducers for superconducting magnet models for the LHC,", CERN-LHC-Project-Report, 1998.

[11] P. Ferracin, E. Todesco, D. Tommasini, and W. Scandale, "Azimuthal coil length and field quality in the main LHC dipoles," Phys. Rev. STAB., submitted for publication. 